\title{
Expectant management of an abdominal pregnancy diagnosed at 18 weeks: a case report
}

\begin{abstract}
A 36 years-old, G2P2 patient presented at $18+2$ weeks of gestation with abdominal pain and vomiting. A transabdominal ultrasound at admission showed a single live fetus according to gestational age, while a transvaginal sonographic examination revealed a normal uterus without an intrauterine gestation. Due to the suspicion of an abdominal pregnancy, the patient was admitted for further study. A magnetic resonance imaging confirms an extrauterine abdominal pregnancy and established placental implantation, blood supply and abdominal organs compromise. Together with the patient and a multidisciplinary team, an expectant management was decided. Serial ultrasound assessments were performed to evaluate fetal condition, as well as serial MRI to exclude any intra-abdominal organ compromise. A laparotomy was performed for fetal extraction at 34 weeks of gestation without complications, leaving the placenta in situ. The patient and newborn had a successful postnatal evolution. This report provides evidence that the expectant management of an abdominal pregnancy diagnosed early in pregnancy is feasible.
\end{abstract}

Keywords: Abdominal pregnancy, Ectopic pregnancy, Advanced extrauterine pregnancy, Expectant management
Volume 9 Issue I - 2018

\author{
Pablo Silva,' Vargas P,',2 Munoz A,' Jofre M,' \\ Briones $\mathrm{P},{ }^{3}$ Riquelme $\mathrm{C},{ }^{4}$ Chavarri M,' Luna \\ $\mathrm{D},{ }^{2} \mathrm{D}$,az $\mathrm{F}^{2}$ Vald?s R, ${ }^{2}$ Ferrer F, ${ }^{1,2}$ Martinovic \\ C,2 Rosas A, ${ }^{2}$ Kusanovic JP', \\ 'Division of Obstetrics and Gynecology, Pontificia Universidad \\ Catolica de Chile, Chile \\ ${ }^{2}$ Department of Obstetrics and Gynecology, Center for \\ Research and Innovation in Maternal-Fetal Medicine (CIMAF), \\ Chile \\ ${ }^{3}$ Division of Surgery, Hospital Sotero del Rio, Chile \\ ${ }^{4}$ Department of Radiology, Hospital S?tero del R
}

Correspondence: Paula Vargas, Designation Center for Research and Innovation in Maternal-Fetal Medicine (CIMAF), Department of Obstetrics and Gynecology, Hospital Stero del Rาo, Santiago,Av. Concha y Toro 3459, Chile, Tel 5699।58I927, Email pasilva@gmail.com

Received: October 05, 2017| Published: January 09, 2018

\section{Introduction}

The prevalence of an ectopic pregnancy is $1-2 \%$, with approximately $95 \%$ of them located in the Fallopian tubes. ${ }^{1}$ An abdominal pregnancy is a very rare form of ectopic pregnancy, which is located in the peritoneal cavity. ${ }^{2}$ The estimated incidence of an abdominal pregnancy is around 1:10.000, representing approximately $1,4 \%$ of all ectopic pregnancies. ${ }^{3-5}$ Potential implantation sites include the uterine serosa, omentum, pelvic wall, broad ligament, posterior cul-de-sac, abdominal organs (i.e spleen, bowel, liver), pelvic vessels, and diaphragm..$^{6-8}$ Given its different forms of clinical presentation, a high index of suspicion is needed to make the diagnosis. Its clinical symptoms include persistent abdominal pain, nausea, vomiting, painful fetal movements, weight loss, vaginal bleeding, and palpation of an abdominal mass distinct from the uterus. ${ }^{9-12}$ Here, we report a successful expectant management of an abdominal pregnancy diagnosed at 18 weeks of gestation.

\section{Case report}

A 36 years-old Hispanic women, G2P2 with two previous term deliveries (two cesarean deliveries), non-sensitized Rh-negative, was admitted at $18+2$ weeks of gestation due to abdominal pain and vomiting. On physical exam, a soft abdomen and stable vital signs were found. A transabdominal ultrasound at admission showed a single live fetus according to gestational age, while a transvaginal sonographic examination revealed a normal uterus without an intrauterine gestation, and a small amount of free fluid. Due to the suspicion of an abdominal pregnancy, the patient was admitted to our High Risk Pregnancy Unit for further evaluation. A magnetic resonance imaging (MRI) was performed and confirmed an extrauterine intraperitoneal gestational sac located in the maternal right flank with a single live fetus inside. The placenta impressed bilobar, located on small bowel, colon, cecum, and part of the mesentery. Computer tomography angiography showed placental blood supply from the right adnexal plexus depending on the hypogastric artery of that side, excluding involvement of great vessels. The case was discussed with the patient and a multidisciplinary team. Since the patient requested an expectant management, she was kept admitted in order to perform serial assessment of the maternal and fetal condition, as well as MRIs for further anatomic assessment, including potential placental invasion of intra-abdominal organs (Figure 1). A double $\mathrm{J}$ stent was installed due to right hydroureteronephrosis, and anti-D inmunoglobulin $(300 \mathrm{mcg})$ was administered at 28 weeks of gestation. Antenatal corticosteroids were administered at 24 weeks of gestation and repeated just before the cesarean section. Delivery was planned at 34 weeks on maternal request due to maternal discomfort. A supraumbilical laparotomy was performed for fetal extraction, and the intraoperative findings revealed an intra-abdominal amniotic sac surrounded by extensive vascular areas with a small a vascular window by which fetal extraction was performed (Figure 2). A female newborn was obtained weighing 2187grs, with an Agar score of 5/8. The placenta was left in situ. The patient has had a successful postoperative first week without any complications. CT scan was performed to evaluate postoperative findings (Figure 3). Human chorionic gonadotropin levels dropped from $115.592 \mathrm{mUI} / \mathrm{mL}$ to $40.840 \mathrm{mUI} / \mathrm{mL}$ during postoperative first week. The need to administer methotrexate was discussed with the Gynecology Oncologist specialists and decided just to follow up with serial images. Patient was discharged at $13^{\text {th }}$ day.

The baby was admitted to Neonatal Intensive Care Unit (NICU) for further observation due to gestational age. During the fifth day in NICU, the newborn developed a necrotizing enterocolitis, with good response to medical treatment and no surgical intervention was required. At second week, the baby was discharged to Intermediate Care Unit. 


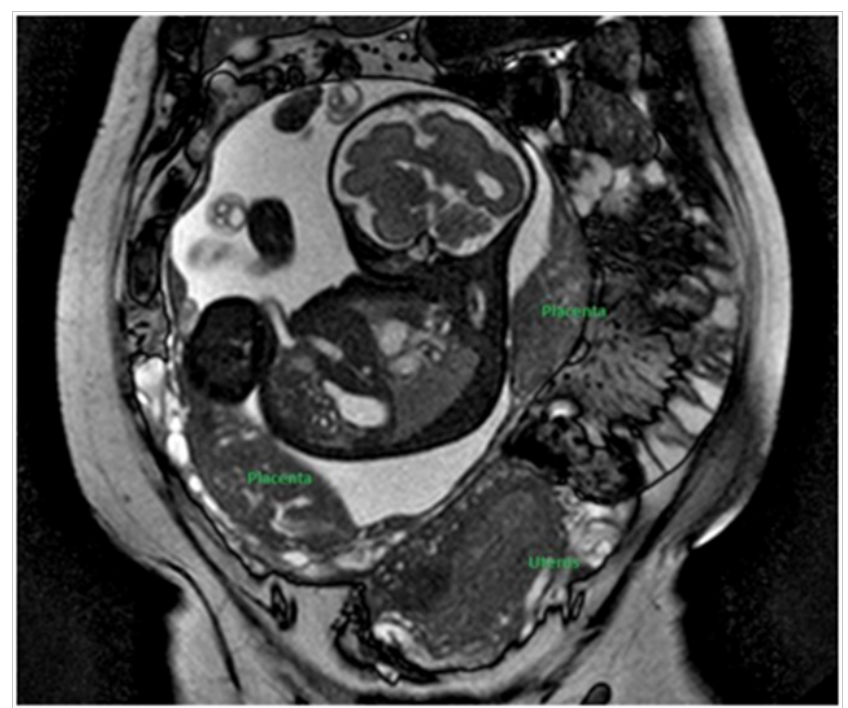

Figure I MRI at 28 weeks showed an extrauterine intraperitoneal gestational sac located in the maternal right flank with a single live fetus inside. The placenta impressed bilobar, located on small bowel, colon, cecum, and part of the mesentery.

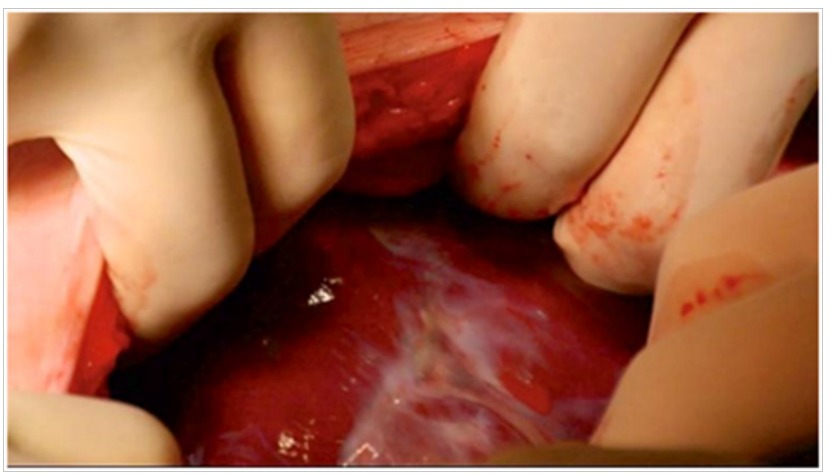

Figure 2 Surgical intrabdominal findings: hyper vascularization of amniotic membranes.

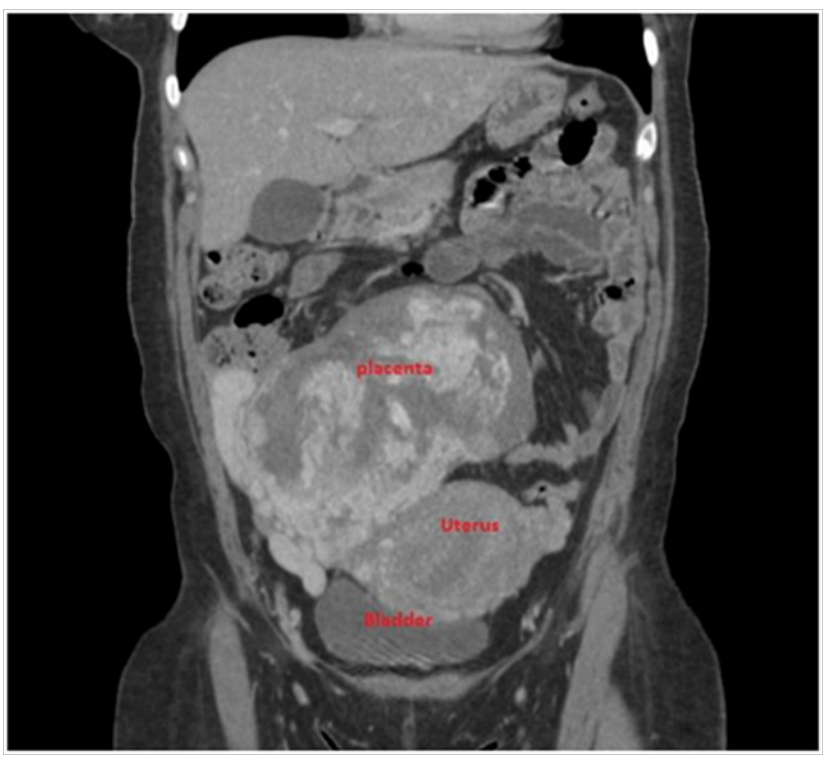

Figure 3 Postnatal CT angiography showed remnant placenta.

\section{Discussion}

Abdominal pregnancy is a very rare entity, representing approximately only $1 \%$ of all ectopic pregnancies. Main risk factors for this condition include tubal damage, pelvic inflammatory disease, endometriosis, assisted reproduction techniques and multiparity. The theories proposed for its etiology are:

a. Due to secondary implantation of a tubal pregnancy aborted, and

b. As a result from an intra-abdominal fertilization. ${ }^{13,14}$

Because clinical signs and symptoms are non-specific, high index of suspicion is needed to make the diagnosis. In a retrospective study, only $45 \%$ of the abdominal pregnancies were diagnosed preoperatively. ${ }^{15}$ The most frequent localizations reported were pouches surrounding uterus, uterine fundus and adnexa, based on a review of abdominal pregnancies diagnosed before 20 weeks. ${ }^{16}$ It is believed that implantation site and blood supply available are key factors to allow the possibility of fetal survival. ${ }^{17,18}$ Ultrasound is the most used diagnostic tool in obstetric practice; nevertheless, it is limited by many factors including maternal obesity, meteorism and operator ability. Despite advances in obstetric ultrasound diagnosis, MRI is a very useful tool to identify placental implantation site, blood supply, and its relationship with great vessels, bowel or other vital structures that could be affected. ${ }^{19-21}$

Fetal survival is exceptional, since the perinatal mortality reported is as high as $80-90 \% .{ }^{22}$ While some authors consider that it is possible an expectant management and wait until fetal lung maturity, others propose that there is a high risk for a life-threatening hemorrhage. ${ }^{23,24}$ In this case, the expectant management until 34 weeks was decided together with a multidisciplinary team without any maternal complication during pregnancy. However, the optimal treatment of an abdominal pregnancy is unknown. Because abdominal pregnancies frequently implant in organs or vascular structures, one of the major issues in delivering an abdominal pregnancy will be hemorrhage control and placental management after delivery. In a review of 225 case reports, mean blood loss was $1450 \mathrm{~mL}$ (range 50-7500 mL) and $25 \%$ of women required blood transfusion. ${ }^{16}$ Among the options available for preoperative preparation are arterial embolization, ureteral catheters insertion, bowel preparation, blood bank assistance for massive transfusion protocols, and a multidisciplinary surgical team available..$^{20,25}$

There is a debate in the literature regarding management and removal of the placenta. Placental removal can be associated to a massive bleeding, and efforts to control the bleeding may lead to organ damage. ${ }^{26,27}$ Leaving the placenta in situ may lead to secondary hemorrhage, abscess formation, adhesions, necrosis, coagulopathy, and need for a second surgical intervention. ${ }^{28,29}$ The partial and complete removal of the placenta have been reported with successful outcome ${ }^{30,31}$ however, if removal of the placenta is not possible due massive vascular invasion, it is recommended to leave the placenta in situ and maintain a close follow-up for involution and early identification of potential complications. Thus, placental management must be individualized and assessed at the operating room, removing it only if it is safe to do..$^{32-34}$ Use of postoperative methotrexate has been described in cases with placenta left in situ, although some authors have proposed that rapid placental destruction leads to accumulation of necrotic debris, favoring bacterial growth. ${ }^{20}$ Rahman et al.${ }^{35}$ reported five patients treated postoperatively with methotrexate. Although they had rapid decline of urinary gonadotropin concentrations, all cases developed intra-abdominal infection and two died. Other authors 
suggest that it could be used at lower doses and with less frequency to prevent fast placental destruction and accumulation of necrotic tissue in excess. ${ }^{36}$

\section{Conclusion}

Here, we present a rare case of an abdominal pregnancy diagnosed at 18 weeks of gestation, followed during pregnancy with expectant management and a favorable maternal and fetal outcome. Serial assessment with MRI allowed us to define placental implantation and vascular involvement preoperatively and also to prepare a multidisciplinary team by the time of surgery. Intraoperatively, an amniotic sac surrounded by significant vascularization was found, and bleeding from the amnioplacental interface was successfully controlled, leaving the placenta in situ.

\section{Acknowledgements}

We would also like to thank to all surgical team from Hospital Sotero del Rio, Santiago, Chile for their support and help for making possible this surgery.

\section{Conflicts of interest}

The authors declare that there is no conflict of interest regarding the publication of this article.

\section{References}

1. Dabiri T, Marroquin GA, Bendek B, et al. Advanced extrauterine pregnancy at 33 weeks with a healthy newborn. Biomed Res Int 2014;2014:102479.

2. Atrash HK, Friede A, Hogue CJ. Abdominal pregnancy in the United States: frequency and maternal mortality. Obstet Gynecol. 1987;69(3 Pt 1):333-337.

3. Dover RW, Powell MC. Management of a primary abdominal pregnancy. Am J Obstet Gynecol. 1995;172(5):1603-1604.

4. Fisch B, Peled Y, Kaplan B, et al. Abdominal pregnancy following in vitro fertilization in a patient with previous bilateral salpingectomy. Obstet Gynecol. 1996;88(4 Pt 2):642-643.

5. Badria L, Amarin Z, Jaradat A, et al. Full-term viable abdomina pregnancy: a case report and review. Arch Gynecol Obstet. $2003 ; 268(4): 340-342$

6. Onan, MA, Turp AB, Saltik A, et al. Primary omental pregnancy: case report. Hum Reprod. 2005;20(3):807-809.

7. Shippey, SH, Bhoola SM, et al. Diagnosis and management of hepatic ectopic pregnancy. Obstet Gynecol. 2007;109(2 Pt2):544-546.

8. Yildizhan R, Kolusari A, Adali F, et al. Primary abdominal ectopic pregnancy: a case report. Cases J. 2009;2: 8485.

9. Mbura JS, Mgaya HN. Advanced abdominal pregnancy in Muhimbili Medical Centre, Tanzania. Int J Gynaecol Obstet. 1986;24(3):169-176.

10. Mutazedian S. Term asymptomatic abdominal pregnancy with good maternal and perinatal outcome: A case report. Ir J Med Sci. 2000;25(12):76-80.

11. Bohiltea R, Radoi V, Tufan C, et al. Abdominal pregnancy - Case presentation. J Med Life. 2015;8(1):49-54.

12. Dubey S, Satodiya M, Garg P, et al. Primary Abdominal Pregnancy: A Case Report. J Clin Diagn Res. 2016;10(11):QD04-QD06.

13. Molinaro TA, Barnhart KT. Ectopic pregnancies in unusual locations Semin Reprod Med. 2007;25(2):123-130.
14. Gudu W, Bekele D. A pre-operatively diagnosed advanced abdominal pregnancy with a surviving neonate: a case report. J Med Case Rep. 2015;9:228.

15. Nkusu Nunyalulendho D, Einterz EM. Advanced abdominal pregnancy: case report and review of 163 cases reported since 1946. Rural Remote Health. 2008;8(4):1087.

16. Poole A, Haas D, Magann EF. Early abdominal ectopic pregnancies: a systematic review of the literature. Gynecol Obstet Invest. 2012;74(4):249-260.

17. Varma R, Mascarenhas L, James D. Successful outcome of advanced abdominal pregnancy with exclusive omental insertion. Ultrasound Obstet Gynecol. 2003;21(2):192-194.

18. Parker VL, Srinivas M. Non-tubal ectopic pregnancy. Arch Gynecol Obstet. 2016;294(1):19-27.

19. Lockhat F, Corr P, Ramphal S, et al. The value of magnetic resonance imaging in the diagnosis and management of extra-uterine abdominal pregnancy. Clin Radiol. 2006;61(3):264-269.

20. Worley KC, Hnat MD, Cunningham FG. Advanced extrauterine pregnancy: diagnostic and therapeutic challenges. Am J Obstet Gynecol. 2008;198(3):e291-e297.

21. Renfroe S, Dajani NK, Pandey T, et al. Role of serial MRI assessment in the management of an abdominal pregnancy. BMJ Case Rep. 2013;pii:bcr2013200495.

22. Cotlar AM. Extrauterine pregnancy: a historical review (3). Curr Surg. 2000;57(5):484-492.

23. Agarwal N, Odejinmi F. Early abdominal ectopic pregnancy: challenges, update and review of current management. The Obstetrician \& Gynaecologist. 2014;16(3):193-198.

24. Gidiri MF, Kanyenze M. Advanced abdominal ectopic pregnancy: lessons from three cases from Zimbabwe and a literature appraisal of diagnostic and management challenges. Womens Health (Lond). 2015;11(3):275-279.

25. Tucker K, Bhardwaj NR, Clark E, et al. Delayed diagnosis and management of second trimester abdominal pregnancy. BMJ Case Rep. 2017; pii:bcr-2017-221433.

26. White RG. Advanced abdominal pregnancy-a review of 23 cases. Ir J Med Sci. 1989;158(4):77-78.

27. Oliver R, Malik M, Coker A, et al. Management of extra-tubal and rare ectopic pregnancies: case series and review of current literature. Arch Gynecol Obstet. 2007;276(2):125-131.

28. Roberts RV, Dickinson JE, Leung Y, et al. Advanced abdominal pregnancy: still an occurrence in modern medicine. Aust $N$ Z J Obstet Gynaecol. 2005;45(6):518-521.

29. Masukume G, Sengurayi E, Muchara A, et al. Full-term abdominal extrauterine pregnancy complicated by post-operative ascites with successful outcome: a case report. J Med Case Rep. 2013;7:10.

30. Baffoe P, Fofie C, Gandau BN. Term abdominal pregnancy with healthy newborn: a case report. Ghana Med J. 2011;45(2):81-83.

31. Brewster EM, Braithwaite EA. Advanced abdominal pregnancy: a case report of good maternal and perinatal outcome. West Indian Med J. 2011;60(5):587-589

32. Aliyu LD, Ashimi AO. A multicentre study of advanced abdominal pregnancy: a review of six cases in low resource settings. Eur J Obstet Gynecol Reprod Biol. 2013;170(1):33-38.

33. Nassali MN, Benti TM, Bandani-Ntsabele M, et al. A case report of an asymptomatic late term abdominal pregnancy with a live birth at 41 weeks of gestation. BMC Res Notes. 2016;9:31. 
34. Hailu FG, Yihunie GT, Essa AA, et al. Advanced abdominal pregnancy, with live fetus and severe preeclampsia, case report. BMC Pregnancy Childbirth. 2017;17(1):243.

35. Rahman MS, Al-Suleiman SA, Rahman J, et al. Advanced abdominal pregnancy- observations in 10 cases. Obstet Gynecol. 1982;59(3):366372.
36. Da Silva BB, de Araujo EP, Cronemberger JN, et al. Primary twin omental pregnancy: report of a rare case and literature review. Fertil Steril. 2008;90(5):e2013-e2005. 